\title{
An Analog Characterization of Elementarily Computable Functions Over the Real Numbers
}

\author{
Olivier Bournez and Emmanuel Hainry \\ LORIA/INRIA, 615 Rue du Jardin Botanique, BP101 \\ 54602 Villers lès Nancy, France \\ \{Olivier.Bournez,Emmanuel.Hainry\}@loria.fr
}

\begin{abstract}
We present an analog and machine-independent algebraic characterization of elementarily computable functions over the real numbers in the sense of recursive analysis: we prove that they correspond to the smallest class of functions that contains some basic functions, and closed by composition, linear integration, and a simple limit schema.

We generalize this result to all higher levels of the Grzegorczyk Hierarchy. Concerning recursive analysis, our results provide machine-independent characterizations of natural classes of computable functions over the real numbers, allowing to define these classes without usual considerations on higher-order (type 2) Turing machines. Concerning analog models, our results provide a characterization of the power of a natural class of analog models over the real numbers.
\end{abstract}

\section{Introduction}

Several approaches have been proposed to model computations over real numbers. Recursive analysis or computable analysis, was introduced by Turing [28], Grzegorczyk [12], Lacombe [15]. Alternative discrete-time computational models have also been investigated: see e.g. [4].

These models concern discrete time computability. Models of machines where the time is continuous can also be considered. The first ever built computers were continuous time machines: e.g. Blaise Pascal's pascaline or Lord Kelvin's model of Differential Analyzer [27], that gave birth to a real machine, built in 1931 at the MIT to solve differential equations [7], and which motivated Shannon's General Purpose Analog Computer (GPAC) model [25], whose computational power was characterized algebraically in terms of solutions of polynomial differential equations $[25,23,16,11]$. Continuous time machines also include analog neural networks [26], hybrid systems [3,5], or theoretical physical models [21,14,10]: see also survey [22].

The relations between all the models are not fully understood. One can say, that the theory of analog computations has not yet experienced the unification that digital discrete time computations have experienced through Turing work and the so-called Church thesis [9,22].

This however becomes a crucial matter since the progress of electronics makes the construction of some of the machines realistic, whereas some models were 
recently proved very (far too?) powerful: using the so-called Zeno's paradox, some models make it possible to compute non-Turing computable functions in a constant time: see e.g. $[17,6,3,14,10]$.

In [17], Moore introduced a class of functions over the reals inspired from the classical characterization of computable functions over integers: observing that the continuous analog of a primitive recursion is a differential equation, Moore proposes to consider the class of $\mathbb{R}$-recursive functions, defined as the the smallest class of functions containing some basic functions, and closed by composition, differential equation solving (called integration), and minimization.

This class of functions, also investigated in $[18,19]$, can be related to GPAC computable functions: see [17], corrected by [11]. The original definitions of this class in [17] suffer from several technical problems, as well as also from some physical realizability problems providing the possibility of using super-Turing "compression tricks".

In his $\mathrm{PhD}$ dissertation, Campagnolo [9] proposes to restrict to the betterdefined subclass $\mathcal{L}$ of $\mathbb{R}$-recursive functions corresponding to the smallest class of functions containing some basic functions and closed by composition and linear integration. Class $\mathcal{L}$ is related to functions elementarily computable over integers in classical recursion theory and functions elementarily computable over the real numbers in recursive analysis (discussed in [30]): any function of class $\mathcal{L}$ is elementarily computable in the sense of recursive analysis, and conversely, any function over the integers computable in the sense of classical recursion theory is the restriction to integers of a function that belongs to $\mathcal{L}[9,8]$.

However, the previous results do not provide a characterization of all functions over the reals that are computable in the sense of recursive analysis. This paper provides one: for functions over the reals of class $\mathcal{C}^{2}$ defined on a product of compact intervals with rational endpoints, $f$ is elementarily computable in the sense of recursive analysis iff it belongs to the smallest class of functions containing some basic functions and closed by composition, linear integration and a simple limit schema. This can be extended to characterize all higher levels of the Grzegorczyk hierarchy: for functions over the reals of class $\mathcal{C}^{2}$ defined on a product of compact intervals with rational endpoints, $f$ is computable in the sense of recursive analysis in level $n \geq 3$ of the Grzegorczyk hierarchy iff $f$ belongs to the smallest class of functions containing some (other) basic functions and closed by composition, linear integration and a simple limit schema.

Concerning analog models, these results have several impacts: first, they contribute to understand analog models, in particular the relations between GPAC computable functions, $\mathbb{R}$-recursive functions, and computable functions in the sense of recursive analysis. Furthermore, they prove that no Super-Turing phenomena can occur for these classes of functions. In particular we have a "robust" class of functions in the sense of $[13,2]$.

Concerning recursive analysis, our theorems provide a purely algebraic and machine independent characterization of elementarily computable functions over the reals. Observe the potential benefits offered by these characterizations com- 
pared to classical definitions of these classes in recursive analysis, involving discussions about higher-order (type 2) Turing machines: see e.g. [29].

In Section 2, we start by some mathematical preliminaries. In Section 3, we recall some notions from classical recursion theory. We present basic definitions of recursive analysis in Section 4. Previous known results are recalled in Section 5. Our characterizations are presented in Section 6. The proofs are given in remaining sections.

\section{Mathematical preliminaries}

Let $\mathbb{N}, \mathbb{Q}, \mathbb{R}, \mathbb{R}^{>0}$ denote the set of natural integers, the set of rational numbers, the set of real numbers, and the set of positive real numbers respectively. Given $x \in \mathbb{R}^{n}$, we write $\vec{x}$ to emphasize that $x$ is a vector.

We will use the following simple mathematical result.

Lemma 1. Let $F: \mathbb{R} \times \mathcal{V} \subset \mathbb{R}^{2} \rightarrow \mathbb{R}^{l}$ be a function of class $^{1} \mathcal{C}^{1}$, and $\beta(x): \mathcal{V} \rightarrow$ $\mathbb{R}$ be some continuous function. Assume that for all $t$ and $x, \frac{\partial^{2} F}{\partial t \partial x}(t, x)$ exists and $\left\|\frac{\partial F}{\partial t}(t, x)\right\| \leq K \exp (-t \beta(x))$, and $\left\|\frac{\partial^{2} F}{\partial t \partial x}(t, x)\right\| \leq K \exp (-t \beta(x))$ for some constant $K>0$.

For all $x \in \mathcal{D}$, where $\mathcal{D}$ is the subset of the $x \in \mathcal{V}$ with $\beta(x)>0, F(t, x)$ has a limit $L(x)$ in $t=+\infty$. Function $L(x)$ is of class $\mathcal{C}^{1}$, and its derivative $L^{\prime}$ is the limit of $\frac{\partial F(t, x)}{\partial x}(t, x)$ in $t=+\infty$. Furthermore

$$
\|F(t, x)-L(x)\| \leq \frac{K \exp (-t \beta(x))}{\beta(x)} \text { and }\left\|\frac{\partial F}{\partial x}(t, x)-L^{\prime}(x)\right\| \leq \frac{K \exp (-t \beta(x))}{\beta(x)} .
$$

The following result ${ }^{2}$, with previous lemma, is a key to provide upper bounds on the growth of functions of our classes (c.f. Lemma 4).

Lemma 2 (Bounding Lemma for Linear Differential Equations [1]). For linear differential equation $\vec{x}^{\prime}=A(t) \vec{x}$, if $A$ is defined and continuous on interval $I=[a, b]$, where $a \leq 0 \leq b$, then, for all $\vec{x}_{0}$, the solution of $\vec{x}^{\prime}=A(t) \vec{x}$ with initial condition $\vec{x}(0)=\vec{x}_{0}$ is defined and unique on I. Furthermore, the solution satisfies

$$
\|\vec{x}(t)\| \leq\left\|\vec{x}_{0}\right\| \exp \left(\sup _{\tau \in[0, t]}\|A(\tau)\| t\right) .
$$

\section{Classical Recursion Theory}

Classical recursion theory deals with functions over integers. Most classes of classical recursion theory can be characterized as closures of a set of basic functions by a finite number of basic rules to build new functions [24,20]: given a set $\mathcal{F}$ of

\footnotetext{
${ }^{1}$ Recall that function $f: \mathcal{D} \subset \mathbb{R}^{k} \rightarrow \mathbb{R}^{l}, k, l \in \mathbb{N}$, is said to be of class $\mathcal{C}^{r}$ if it is $r$-times continuously differentiable on $\mathcal{D}$.

${ }^{2}$ As it was already the case in Campagnolo's Dissertation.
} 
functions and a set $\mathcal{O}$ of operators on functions (an operator is an operation that maps one or more functions to a new function), $[\mathcal{F} ; \mathcal{O}]$ will denote the closure of $\mathcal{F}$ by $\mathcal{O}$.

Proposition 1 (Classical settings: see e.g. [24,20]). Let $f$ be a function from $\mathbb{N}^{k}$ to $\mathbb{N}$ for $k \in \mathbb{N}$. Function $f$ is

- elementary iff it belongs to $\mathcal{E}=[0, S, U,+, \ominus$; COMP, BSUM, BPROD];

- in class $\mathcal{E}_{n}$ of the Grzegorczyk Hierarchy $(n \geq 3)$ iff it belongs to $\mathcal{E}_{n}=$ $\left[0, S, U,+, \ominus, E_{n-1}\right.$; COMP, BSUM, BPROD];

- primitive recursive iff it belongs to $\mathcal{P} \mathcal{R}=[0, U, S ; \mathrm{COMP}, \mathrm{REC}]$;

- recursive iff it belongs to Rec $=[0, U, S$; COMP, REC, MU $]$.

A function $f: \mathbb{N}^{k} \rightarrow \mathbb{N}^{l}$ is elementary (resp: primitive recursive, recursive) iff its projections are elementary (resp: primitive recursive, recursive).

The base functions $0,\left(U_{i}^{m}\right)_{i, m \in \mathbb{N}}, S,+, \ominus$ and the operators COMP, BSUM, BPROD, REC, MU are given by

1. $0: \mathbb{N} \rightarrow \mathbb{N}, 0: n \mapsto 0 ; U_{i}^{m}: \mathbb{N}^{m} \rightarrow \mathbb{N}, U_{i}^{m}:\left(n_{1}, \ldots, n_{m}\right) \mapsto n_{i} ; S: \mathbb{N} \rightarrow$ $\mathbb{N}, S: n \mapsto n+1 ;+: \mathbb{N}^{2} \rightarrow \mathbb{N},+:\left(n_{1}, n_{2}\right) \mapsto n_{1}+n_{2} ; \ominus: \mathbb{N}^{2} \rightarrow \mathbb{N}$, $\ominus:\left(n_{1}, n_{2}\right) \mapsto \max \left(0, n_{1}-n_{2}\right)$;

2. BSUM : bounded sum. Given $f, h=\operatorname{BSUM}(f)$ is defined by $h:(\vec{x}, y) \mapsto$ $\sum_{z<y} f(\vec{x}, z)$; BPROD : bounded product. Given $f, h=\operatorname{BPROD}(f)$ is defined by $h:(\vec{x}, y) \mapsto \prod_{z<y} f(\vec{x}, z)$;

3. COMP : composition. Given $f$ and $g, h=\operatorname{COMP}(f, g)$ is defined as the function verifying $h(\vec{x})=g(f(\vec{x}))$;

4. REC : primitive recursion. Given $f$ and $g, h=\operatorname{REC}(f, g)$ is defined as the function verifying $h(\vec{x}, 0)=f(\vec{x})$ and $h(\vec{x}, n+1)=g(\vec{x}, n, h(\vec{x}, n))$.

5. MU : minimization. The minimization of $f$ is $h: \vec{x} \mapsto \inf \{y: f(\vec{x}, y)=0\}$.

Functions $E_{n}$, involved in the definition of the classes $\mathcal{E}_{n}$ of the Grzegorczyk Hierarchy, are defined by induction as follows (when $f$ is a function, $f^{[d]}$ denotes its d-th iterate: $\left.f^{[0]}(\vec{x})=x, f^{[d+1]}(\vec{x})=f\left(f^{[d]}(\vec{x})\right)\right)$ :

1. $E_{0}(x, y)=x+y, E_{1}(x, y)=(x+1) \times(y+1), E_{2}(x)=2^{x}$;

2. $E_{n+1}(x)=E_{n}^{[x]}(1)$ for $n \geq 2$.

We have $\mathcal{E} \subseteq \mathcal{P} \mathcal{R} \subseteq \mathcal{R} e c$, and the inclusions are known to be strict $[24,20]$. It is also known that $\mathcal{E}_{3}=\mathcal{E}$ and $\mathcal{P} \mathcal{R}=\cup_{i} \mathcal{E}_{i}[24,20]$. If $\operatorname{TIME}(t)$ and $\operatorname{SPACE}(t)$ denote the classes of functions that are computable with time and space $t$, then, for all $n \geq 3, \mathcal{E}_{n}=\operatorname{TIME}\left(\mathcal{E}_{n}\right)=\operatorname{SPACE}\left(\mathcal{E}_{n}\right)$, and $\mathcal{P} \mathcal{R}=\operatorname{TIME}(\mathcal{P} \mathcal{R})=$ $\operatorname{SPACE}(\mathcal{P} \mathcal{R})[24,20]$. $\mathcal{P} \mathcal{R}$ corresponds to functions computable using loop programs. $\mathcal{E}$ corresponds to computable functions bounded by some iterate of the exponential function $[24,20]$.

In classical computability, more general objects than functions over the integers can be considered, in particular functionals, i.e. functions $\Phi:\left(\mathbb{N}^{\mathbb{N}}\right)^{m} \times \mathbb{N}^{k} \rightarrow$ $\mathbb{N}^{l}$. A functional will be said to be elementary (respectively. $\mathcal{E}_{n}$, primitive recursive, recursive) when it belongs to the corresponding ${ }^{3}$ class.

\footnotetext{
${ }^{3}$ Formally, a function $f$ over the integers can be considered as functional $\bar{f}$ :
} $\left(V_{1}, \ldots, V_{m}, \vec{n}\right) \mapsto f(\vec{n})$. Similarly, an operator $O p$ on functions $f_{1}, \ldots, f_{m}$ 


\section{Computable Analysis}

The idea sustaining Computable analysis, also called recursive analysis, is to define computable functions over real numbers by considering functionals over fast-converging sequences of rationals $[28,15,12,29]$.

Formally, assume that a representation of rational numbers by integers is fixed $^{4}$ : let $\nu_{\mathbb{Q}}(r)$ be the rational represented by integer $r$. A product $\mathcal{C}=\left[a_{1}, b_{1}\right] \times$ $\ldots \times\left[a_{k}, b_{k}\right]$ of compact intervals with rational endpoints can be encoded by an integer $\nu(\mathcal{C})$ encoding the list $\left\langle\nu_{\mathbb{Q}}\left(a_{1}\right), \nu_{\mathbb{Q}}\left(b_{1}\right), \ldots, \nu_{\mathbb{Q}}\left(a_{k}\right), \nu_{\mathbb{Q}}\left(b_{k}\right)\right\rangle$.

A sequence of integers $\left(x_{i}\right) \in \mathbb{N}^{\mathbb{N}}$ represents a real number $x$ if it converges quickly toward $x$ (denoted by $\left(x_{i}\right) \rightsquigarrow x$ ) in the following sense: $\forall i,\left|\nu_{\mathbb{Q}}\left(x_{i}\right)-x\right|<$ $\exp (-i)$. For $X=\left(\left(x_{1}\right), \ldots,\left(x_{k}\right)\right) \in\left(\mathbb{N}^{\mathbb{N}}\right)^{k}, \vec{x} \in \mathbb{R}^{k}$, we write $X \rightsquigarrow \vec{x}$ for $\left(x_{i}\right) \rightsquigarrow U_{i}^{k}(\vec{x})$ for $i=1, \ldots, k$.

Definition 1 (Recursive analysis). A function $f: \mathcal{D} \rightarrow \mathbb{R}$, where $\mathcal{D} \subset \mathbb{R}^{k}$ is a product of compact intervals with rationals endpoints, is said to be computable (in the sense of recursive analysis) if there exists a recursive functional $\phi:\left(\mathbb{N}^{\mathbb{N}}\right)^{k} \times$ $\mathbb{N} \rightarrow \mathbb{N}$ such that for all $\vec{x} \in \mathcal{D}$, for all $X \in\left(\mathbb{N}^{\mathbb{N}}\right)^{k}$, we have $(\phi(X, j))_{j} \rightsquigarrow f(\vec{x})$ whenever $X \rightsquigarrow \vec{x}$.

A function $f: \mathcal{D} \rightarrow \mathbb{R}$, where $\mathcal{D} \subset \mathbb{R}^{k}$ is not necessarily compact, is said to be computable if there exists a recursive functional $\phi:\left(\mathbb{N}^{\mathbb{N}}\right)^{k} \times \mathbb{N}^{2} \rightarrow \mathbb{N}$ such that for all product $\mathcal{C}$ of compact intervals with rational endpoints included in $\mathcal{D}$, $\forall \vec{x} \in \mathcal{C}$, for all $X \in\left(\mathbb{N}^{\mathbb{N}}\right)^{k}$, we have $(\phi(X, \nu(\mathcal{C}), j))_{j} \rightsquigarrow f(\vec{x})$ whenever $X \rightsquigarrow \vec{x}$.

A function $f: \mathcal{D} \rightarrow \mathbb{R}^{l}$, with $l>1$, is said to be computable if all its projections are.

A function $f$ will be said to be elementarily (respectively $\mathcal{E}_{n}$ ) computable whenever the corresponding functional $\Phi$ is. The class of elementarily (respectively $\mathcal{E}_{n}$ ) computable functions over the reals will be denoted by $\mathcal{E}(\mathbb{R}$ ) (resp. $\left.\mathcal{E}_{n}(\mathbb{R})\right)$. Observe that elementarily computable functions were discussed in [30].

\section{Real-recursive and recursive functions}

Following the original ideas from [17], but avoiding the minimization schema, Campagnolo proposed in [9] to consider the following class, built in analogy with elementarily computable functions over the integers (a real extension of a function $f: \mathbb{N}^{k} \rightarrow \mathbb{N}^{l}$ is a function $\tilde{f}$ from $\mathbb{R}^{k}$ to $\mathbb{R}^{l}$ whose restriction to $\mathbb{N}^{k}$ is $f)$.

over the integers can be extended to $\overline{O p}\left(F_{1}, \ldots, F_{m}\right):\left(V_{1}, \ldots, V_{m}, \vec{n}\right) \mapsto$ $O p\left(F_{1}\left(V_{1}, \ldots, V_{m},.\right), \ldots, F_{m}\left(V_{1}, \ldots, V_{m},.\right)\right)(\vec{n})$. We will still (abusively) denote by $\left[f_{1}, \ldots, f_{p} ; O_{1}, \ldots, O_{q}\right]$ for the smallest class of functionals that contains basic functions $\overline{f_{1}}, \ldots, \overline{f_{p}}$, plus the functionals $M_{a p_{i}}:\left(V_{1}, \ldots, V_{m}, n\right) \rightarrow\left(V_{i}\right)_{n}$, the nth element of sequence $V_{i}$, and which is closed by the operators $\overline{O_{1}}, \ldots, \overline{O_{q}}$. For example, a functional will be said to be elementary iff it belongs to $\mathcal{E}=$ $[$ Map $, \overline{0}, \bar{S}, \bar{U}, \mp, \bar{\ominus} ; \overline{\mathrm{COMP}}, \overline{\mathrm{BSUM}}, \overline{\mathrm{BPROD}}]$.

${ }^{4}$ We will assume that in this representation, the basic functions on rationals,,$+- \times$, / are elementarily computable. 
Definition $2([\mathbf{9}, \mathbf{8}])$. Let $\mathcal{L}$ and $\mathcal{L}_{n}$ be the classes of functions $f: \mathbb{R}^{k} \rightarrow$ $\mathbb{R}^{l}$, for some $k, l \in \mathbb{N}$, defined by $\mathcal{L}=\left[0,1,-1, \pi, U, \theta_{3} ; \mathrm{COMP}, \mathrm{LI}\right]$ and $\mathcal{L}_{n}=$ $\left[0,1,-1, \pi, U, \theta_{3}, \overline{E_{n-1}}\right.$; COMP, LI $]$ where the base functions $0,1,-1, \pi,\left(U_{i}^{m}\right)_{i, m \in \mathbb{N}}$, $\theta_{3}, \overline{E_{n}}$ and the schemata COMP and LI are defined as follows:

1. $0,1,-1, \pi$ are the corresponding constant functions; $U_{i}^{m}: \mathbb{R}^{m} \rightarrow \mathbb{R}$ are, as in the classical settings, projections: $U_{i}^{m}:\left(x_{1}, \ldots, x_{m}\right) \mapsto x_{i}$;

2. $\theta_{3}: \mathbb{R} \rightarrow \mathbb{R}$ is defined as $\theta_{3}: x \mapsto x^{3}$ if $x \geq 0,0$ otherwise.

3. $\overline{E_{n}}$ : for $n \geq 3$, let $\overline{E_{n}}$ denote a monotone real extension of the function $\exp _{n}$ over the integers defined inductively by $\exp _{2}(x)=2^{x}, \exp _{i+1}(x)=\exp _{i}^{[x]}(1)$.

4. COMP: composition is defined as in the classical settings: Given $f$ and $g$, $h=\operatorname{COMP}(f, g)$ is the function verifying $h(\vec{x})=g(f(\vec{x}))$;

5. LI: linear integration. From $g$ and $h, \mathrm{LI}(g, h)$ is the maximal solution of the linear differential equation $\frac{\partial f}{\partial y}(\vec{x}, y)=h(\vec{x}, y) f(\vec{x}, y)$ with $f(\vec{x}, 0)=$ $g(\vec{x})$.

In this schema, if $g$ goes to $\mathbb{R}^{n}, f=\operatorname{LI}(g, h)$ also goes to $\mathbb{R}^{n}$ and $h(\vec{x}, y)$ is a $n \times n$ matrix with elements in $\mathcal{L}$.

These classes contain functions $i d: x \mapsto x, \sin , \cos , \exp ,+, \times, x \mapsto r$ for all rational $r$, as well as for all $f \in \mathcal{L}$, or $f \in \mathcal{L}^{*}$, its primitive function $F$ equal to $\overrightarrow{0}$ at $\overrightarrow{0}$, denoted by $\int(f)$. Indeed, function $i d$ is given by $\operatorname{LI}(1,0)$. Function $\Theta$ : $t \mapsto(\sin (t), \cos (t))$ can be defined by LI $\left(\left[\begin{array}{l}0 \\ 1\end{array}\right],\left[\begin{array}{cc}0 & 1 \\ -1 & 0\end{array}\right]\right)$. Project this function on each of its two variables to get sinus and cosinus function. Function exp is given by $\operatorname{LI}(1, i d)$. Addition is given by $x+0=x, \frac{\partial x+y}{\partial y}=1$. Multiplication is given by $x \times 0=0, \frac{\partial x \times y}{\partial y}=x$. $\int(f)$ can be defined by $\left(\begin{array}{c}F \\ 1\end{array}\right)=\mathrm{LI}\left(\left[\begin{array}{l}0 \\ 1\end{array}\right],\left[\begin{array}{ll}0 & f \\ 0 & 0\end{array}\right]\right)$. Given $p, q \in \mathbb{N}$ with $q>0$, Function $x \mapsto p$, is $1+1+\ldots+1$, function $x \mapsto x^{q-1}$ is $x \times \ldots \times x$, and $p \times \int\left(x \mapsto x^{q-1}\right)$ is $x \mapsto p x^{q} / q$ whose value in 1 is $p / q$.

Proposition 2 ([8]). All functions from $\mathcal{L}$ are continuous, defined everywhere, and of class $\mathcal{C}^{2}$.

The previous classes can be partially related to classes $\mathcal{E}(\mathbb{R})$ and $\mathcal{E}_{n}(\mathbb{R})$ :

Proposition $3([9,8])$.

- 1. $\mathcal{L} \subset \mathcal{E}(\mathbb{R})$ : any function from $\mathcal{L}$ is elementarily computable over real numbers.

2. "E् $\subset \mathcal{L}$ ": any elementarily computable function over the integers, has a real extension that belongs to $\mathcal{L}$.

- 1. $\mathcal{L}_{n} \subset \mathcal{E}_{n}(\mathbb{R})$ : any function from $\mathcal{L}_{n}$ is $\mathcal{E}_{n}$-computable.

2. " $\mathcal{E}_{n} \subset \mathcal{L}_{n}$ ": any $\mathcal{E}_{n}$-computable function over the integers, has a real extension that belongs to $\mathcal{L}_{n}$.

Although Proposition 3 gives the inclusions $\mathcal{L} \subset \mathcal{E}(\mathbb{R})$ and $\mathcal{L}_{n} \subset \mathcal{E}_{n}(\mathbb{R})$, it fails to characterize completely $\mathcal{E}(\mathbb{R})$ and $\mathcal{E}_{n}(\mathbb{R})$ : these inclusions are strict. Indeed, $x \mapsto 1 / x$ is elementarily computable while Proposition 2 says that all functions from $\mathcal{L}$ are defined everywhere. A similar argument works for $\mathcal{E}_{n}(\mathbb{R})$. We conjecture the inclusions to be strict even when restricting to total functions. 


\section{Real-recursive and recursive functions revisited}

We now propose to consider new classes of functions that we will prove to correspond precisely to $\mathcal{E}(\mathbb{R})$ and $\mathcal{E}_{n}(\mathbb{R})$.

First, we modify a little bit the composition schema, since (non-total) elementarily computable functions are not stable by composition.

Definition 3 (COMP schema). Given $f$, $g$, if there is a product of closed intervals ${ }^{5} C$ with rational or infinite endpoints with Range $(f) \subset C \subset \operatorname{Domain}(g)$, then function $\operatorname{COMP}(f, g)$ is defined. It is defined by $\operatorname{COMP}(f, g): \vec{x} \mapsto$ $g(f(\vec{x}))$ on all $\vec{x}$ where $f(\vec{x})$ and $g(f(\vec{x}))$ exist.

Now, we suggest to add a limit operator denoted by LIM, inspired by Lemma 1 : a polynomial $\beta$ over $\mathbb{R}$ is a function of the form $\beta: \mathbb{R} \rightarrow \mathbb{R}, \beta: x \mapsto \sum_{i=0}^{n} a_{i} x^{i}$ for some $a_{0}, \ldots, a_{n} \in \mathbb{R}$.

Definition 4 (LIM schema). Let $f: \mathbb{R} \times \mathcal{D} \subset \mathbb{R}^{2} \rightarrow \mathbb{R}$, and $\beta: \mathcal{D} \rightarrow \mathbb{R} a$ polynomial with the following hypothesis: there exists a constant $K$ such that for all $t, x,\left\|\frac{\partial f}{\partial t}(t, x)\right\| \leq K \exp (-t \beta(x)), \frac{\partial^{2} f}{\partial t \partial x}(t, x)$ exists, and $\left\|\frac{\partial^{2} f}{\partial t \partial x}(t, x)\right\| \leq$ $K \exp (-t \beta(x))$.

Then, for every interval $I \subset \mathbb{R}$ on which $\beta(x)>0, F=\operatorname{LIM}(f, \beta)$ is defined as the function $F: I \rightarrow \mathbb{R}$, with $F(x)=\lim _{t \rightarrow+\infty} f(t, x)$, under the condition that it is of class $^{6} \mathcal{C}^{2}$.

We are ready to define our classes:

Definition 5 (Classes $\mathcal{L}^{*}, \mathcal{L}_{n}^{*}$ ). The class $\mathcal{L}^{*}$, and $\mathcal{L}_{n}^{*}$, for $n \geq 3$, of functions from $\mathbb{R}^{k}$ to $\mathbb{R}^{l}$, for $k, l \in \mathbb{N}$, are following classes:

$-\mathcal{L}^{*}=\left[0,1,-1, U, \theta_{3} ; \mathrm{COMP}, \mathrm{LI}, \mathrm{LIM}\right]$.

$-\mathcal{L}_{n}^{*}=\left[0,1,-1, U, \theta_{3}, \overline{E_{n-1}} ;\right.$ COMP, LI, LIM $]$.

Example 1. Previous classes can easily be shown stable by the primitive operator that sends a function $f$ to its primitive $\int(f)$ equal to $\overrightarrow{0}$ at $\overrightarrow{0}$.

Class $\mathcal{L}^{*}$ also includes some non-total functions, in particular the function $\frac{1}{x}$ : $\mathbb{R}^{>0} \rightarrow \mathbb{R}, \frac{1}{x}: x \mapsto \frac{1}{x}$ : indeed, $\int(\exp (-t x))$ is function $E:(t, x) \mapsto \frac{(1-\exp (-t x))}{x}$ for $x \neq 0, t$ for $x=0$ (of class $\mathcal{C}^{k}$ for all $k$ ). Now $\frac{1}{x}=\operatorname{LIM}(E, i d)$.

Proposition 4. $\mathcal{L} \subsetneq \mathcal{L}^{*}, \mathcal{L}_{n} \subsetneq \mathcal{L}_{n}^{*}$ for all $n \geq 3$.

Proof. The function $x \mapsto \pi$ is actually in $\mathcal{L}^{*}$. Indeed, from $x \mapsto \frac{1}{1+x^{2}}$ in the class, we have $\arctan x=\int\left(\frac{1}{1+x^{2}}\right)$, and $\pi=4 \arctan (1)$. Observing that our composition schema for total functions subsumes the composition schema of class $\mathcal{L}$, the result follows.

${ }^{5}$ That can be $\mathbb{R}^{k}$ when $g$ is total.

${ }^{6}$ If $f$ is of class $\mathcal{C}^{1}$, function $F$ exists and is at least of class $\mathcal{C}^{1}$ by Lemma 1 . 
The main results of this paper are the following (proved in following two sections):

Theorem 1 (Characterization of $\mathcal{E}(\mathbb{R})$ ). Let $f: \mathcal{D} \subset \mathbb{R}^{k} \rightarrow \mathbb{R}^{l}$ be some function over the reals of class $\mathcal{C}^{2}$, with $\mathcal{D}$ product of compact intervals with rational endpoints. $f$ is in $\mathcal{E}(\mathbb{R})$ iff it belongs to $\mathcal{L}^{*}$.

Theorem 2 (Characterization of $\mathcal{E}_{n}(\mathbb{R})$ ). Let $f: \mathcal{D} \subset \mathbb{R}^{k} \rightarrow \mathbb{R}^{l}$ be some function over the reals of class $\mathcal{C}^{2}$, with $\mathcal{D}$ product of compact intervals with rational endpoints. Let $n \geq 3$. $f$ is in $\mathcal{E}_{n}(\mathbb{R})$ iff it belongs to $\mathcal{L}_{n}^{*}$.

\section{Upper bounds}

We now prove the upper bound $\mathcal{L}^{*} \subset \mathcal{E}(\mathbb{R})$. As one may expect, this direction of the proof has many similarities with the proof $\mathcal{L} \subset \mathcal{E}$ in [9,8]: main differences lie in the presence of non-total functions and of schema LIM.

A structural induction shows:

Lemma 3. All functions from $\mathcal{L}^{*}$ are of class $\mathcal{C}^{2}$ and defined on a domain of the form $I_{1} \times I_{2} \ldots \times I_{k}$ where each $I_{i}$ is an interval.

We propose to introduce the following notation: given $a \in \mathbb{R}$, let $\rho_{a}$ be the function $x \mapsto \frac{1}{x-a}$. Let $\rho_{+\infty}$ and $\rho_{-\infty}$ be the function identity $x \mapsto x$.

Given $I$ real interval with bounds $a, b \in \mathbb{R} \cup\{-\infty,+\infty\}, \rho_{I}(x)=\left|\rho_{a}(x)\right|+$ $\left|\rho_{b}(x)\right|$. For $\mathcal{D}=I_{1} \times I_{2} \ldots \times I_{k}$, let $\rho_{\mathcal{D}}(x)=\rho_{I_{1}}\left(U_{1}^{k}(x)\right)+\ldots+\rho_{I_{k}}\left(U_{k}^{k}(x)\right)$. In any case, $\rho_{\mathcal{D}}(x)$ is elementarily computable and grows to $+\infty$ when $x$ gets close to a bound of domain $\mathcal{D}$.

The following Lemma is an extension of a Lemma of [9,8] (it is proved by structural induction using Lemma 1 for schema LIM, Lemma 2 for schema LI, plus the fact that it is always possible to assume that the degree of a product or a sum of two functions $f$ and $g$ is less than the maximum of their degrees).

Lemma 4. Let $f: \mathcal{D} \subset \mathbb{R}^{k} \rightarrow \mathbb{R}^{l}$ be a function of $\mathcal{L}^{*}$. There exist some integer $d$, and some constants $A$ and $B$ such that for all $\vec{x} \in \mathcal{D},\|f(\vec{x})\| \leq$ $A \exp ^{[d]}\left(B \rho_{\mathcal{D}}(\vec{x})\right)$. Call the smallest such integer $d$ the degree of $f$. All the partial derivatives of $f$ also have a finite degree.

We are ready to prove the upper bound.

Proposition 5. $\mathcal{L}^{*} \subseteq \mathcal{E}(\mathbb{R})$.

Proof. - The basic functions $0,1,-1, U, \theta_{3}$ are easily shown elementarily computable.

- When $h=\operatorname{COMP}(f, g), f$ and $g$ elementarily computable, then $h$ is also elementarily computable: indeed, there exists some closed set $F$ with Range $(f)$ $\subset F \subset \operatorname{Domain}(g)$. Adapting the constructions in [29], given a product of compact intervals $\mathcal{C}$ with rational endpoints included in Domain $(f)$, we can compute elementarily a product of compact intervals $\mathcal{C}^{\prime}$ with rational endpoints with $f(\mathcal{C}) \subset \mathcal{C}^{\prime}$. Now, for $x \in \mathcal{C}$, compose the functional that computes $g$ on $\mathcal{C}^{\prime} \cap F$ with the one that computes $f$ on $\mathcal{C}$. 
- Let $g=\operatorname{LIM}(f, \beta)$, with $f$ computed by elementary functional $\phi$. We give the proof for $f$ defined on $\mathbb{R} \times \mathcal{C}$ where $\mathcal{C}$ is a compact interval of $\mathbb{R}$. The general case is easy to obtain.

Let $x \in \mathbb{R}$, with $\beta(x)>0$. Since $\beta(x)$ is a polynomial, $1 / \beta(x)$ can be bounded elementarily by some computable integer $N$ in some computable neighborhood of $x$.

Let $\left(x_{n}\right) \rightsquigarrow x$. For all $i, j \in \mathbb{N}$, if we write $(i)$ for the constant sequence $k \mapsto i$, we have $\left|\nu_{\mathbb{Q}}\left(\phi\left((i),\left(x_{n}\right), j\right)\right)-f(i, x)\right|<\exp (-j)$.

By Lemma 1, we have $\|f(i, x)-g(x)\| \leq \frac{K \exp (-\beta(x) i)}{\beta(x)} \leq K N \exp (-\beta(x) i)$. Hence, $\left|\nu_{\mathbb{Q}}\left(\phi\left((i),\left(x_{n}\right), j\right)\right)-g(x)\right|<\exp (-j)+K N \exp (-\beta(x) i)$.

If we take $j^{\prime}=j+1, i^{\prime}=N(j+1+\lceil\ln (K N)\rceil)$, we have $\exp \left(-j^{\prime}\right) \leq$ $\exp (-j) / 2$, and $K N \exp \left(-\beta(x) i^{\prime}\right) \leq \exp (-j) / 2$. Hence $g$ is computed by the functional $\psi:\left(\left(x_{n}\right), j\right) \mapsto \phi\left((N(j+1+\lceil\ln (K N)\rceil)),\left(x_{n}\right), j+1\right)$.

- Let $f=\operatorname{LI}(g, h)$. The proof for this case is very similar to $[9,8]$.

This ends the proof.

Replacing in previous proofs the bounds of Lemma 4 by bounds of type $\|f(\vec{x})\| \leq A \bar{E}_{n-1}^{[d]}\left(B \rho_{\mathcal{D}}(\vec{x})\right)$, one can also obtain.

Proposition 6. $\forall n \geq 3, \mathcal{L}_{n}^{*} \subseteq \mathcal{E}_{n}(\mathbb{R})$.

\section{Lower bounds}

We will now consider the opposite inclusion: $\mathcal{E}(\mathbb{R}) \subseteq \mathcal{L}^{*}$, proved for functions of class $\mathcal{C}^{2}$ on compact domains with rational endpoints.

Let $\epsilon>0$ be some real. We write $\mathbb{N} \epsilon$ for the set of reals of the form $i \epsilon$ for some integer $i$. Given $y \in \mathbb{R}$, write $\lfloor y\rfloor_{\epsilon}$ for the unique $j \epsilon$ with $j$ integer and $y \in[j \epsilon, j \epsilon+\epsilon)$.

Lemma 5. Let $\epsilon: \mathbb{R} \rightarrow \mathbb{R}$ be some decreasing elementarily computable function, with $\epsilon(x)>0$ for all $x$ and going to 0 when $x$ goes to $+\infty$. Write $\epsilon_{i}$ for $\epsilon(\lfloor i\rfloor)$.

Given $f: \mathbb{R}^{2} \rightarrow \mathbb{R}^{l}$ in $\mathcal{L}^{*}$, there exists $F: \mathbb{R}^{2} \rightarrow \mathbb{R}^{l}$ in $\mathcal{L}^{*}$ with the following properties:

- For all $i \in \mathbb{N}, x \in \mathbb{N} \epsilon_{i}, F(i, x)=f(i, x)$

- For all $i \in \mathbb{N}, x \in \mathbb{R},\left\|F(i, x)-f\left(i,\lfloor x\rfloor_{\epsilon_{i}}\right)\right\| \leq\left\|f\left(i,\lfloor x\rfloor_{\epsilon_{i}}+\epsilon_{i}\right)-f\left(i,\lfloor x\rfloor_{\epsilon_{i}}\right)\right\|$

- For all $i \in \mathbb{R}, x \in \mathbb{R},\left\|\frac{\partial F}{\partial i}(i, x)\right\| \leq 5\left\|f\left(\lfloor i+1\rfloor,\lfloor x\rfloor \epsilon_{\epsilon_{i}}\right)-f\left(\lfloor i\rfloor,\lfloor x\rfloor \epsilon_{\epsilon_{i}}\right)\right\|+$ $25\left\|f\left(\lfloor i\rfloor,\lfloor x\rfloor_{\epsilon_{i}}+\epsilon_{i}\right)-f\left(\lfloor i\rfloor,\lfloor x\rfloor_{\epsilon_{i}}\right)\right\|+25 \| f\left(\lfloor i+1\rfloor,\lfloor x\rfloor_{\epsilon_{i+1}}+\epsilon_{i+1}\right)-f(\lfloor i+$ $\left.1\rfloor,\lfloor x\rfloor_{\epsilon_{i+1}}\right) \|$.

Proof (Sketch). Let $\zeta=\frac{3 \pi}{2}$. Let $\omega: x \mapsto \zeta \theta_{3}(\sin (2 \pi x)) . \forall i, \int_{i}^{i+1} \omega=1$ and $\omega$ is equal to 0 on $\left[i+\frac{1}{2}, i+1\right]$ for every $i \in \mathbb{N}$. Let $\Omega=\int(\omega)$ its primitive, and int : $x \mapsto \Omega\left(x-\frac{1}{2}\right)$. The function int is similar to the integer part: $\forall i$, $\forall x \in\left[i, i+\frac{1}{2}\right], \operatorname{int}(x)=i=\lfloor x\rfloor$. Let $\Delta(i, x)=f(i, x+\epsilon(i))-f(i, x)$.

Let $G$ be the solution of the linear differential equation $G(i, 0)=f(i, 0)$, $\frac{\partial G}{\partial x}(i, x)=\frac{\omega(x / \epsilon(i))}{\epsilon(i)} \Delta(i, \epsilon(i) \operatorname{int}(x / \epsilon(i)))$. An easy induction on $j$ then shows that $G(i, j \epsilon(i))=f(i, j \epsilon(i))$ for all integer $j$. 
Then, let $\Delta^{\prime}(i, x)=G(i+1, x)-G(i, x)$.

Let $F$ be the solution of the linear differential equation $F(0, x)=G(0, x)$, $\frac{\partial F}{\partial i}=\omega(i) \Delta^{\prime}(\operatorname{int}(i), x)$. By induction, we have $F(i, x)=f(i, x)$ for all $i \in \mathbb{N}$, $x \in \mathbb{N} \epsilon_{i}$.

Some technical computations allow to conclude that function $F$ satisfies all the claims.

We are now ready to prove the missing inclusion of Theorem 1.

Proposition 7. Let $f: \mathcal{D} \subset \mathbb{R}^{k} \rightarrow \mathbb{R}^{l}$ be some function over the reals of class $\mathcal{C}^{2}$, with $\mathcal{D}$ product of compact intervals with rational endpoints. If $f$ is $\mathcal{E}(\mathbb{R})$, then it belongs to $\mathcal{L}^{*}$.

Proof. We give the proof for a function $f$ defined on interval $[0,1]$ to $\mathbb{R}$. The general case is easy to obtain.

Since $f^{\prime \prime}$ is continuous on a compact set, $f^{\prime \prime}$ is bounded by some constant $M$. By mean value theorem, we have $\left|f^{\prime}(x)-f^{\prime}(y)\right| \leq M|x-y|$ for all $x, y$.

Given $i$, consider $n$ with $\exp (n) \exp (-i) \geq 4 M$ and $\exp (-n) \leq 1 / 4$. For all $j$, consider $x_{j}=j \exp (-n)$, so that for all $x, y \in\left[x_{j}, x_{j+1}\right]$ we have $\left|f^{\prime}(x)-f^{\prime}(y)\right| \leq$ $\exp (-i) / 4$.

For all $j$, let $y_{j}$ be some rational number at most $\exp (-i) / 2$ far from $f\left(x_{j}\right)$ and $z_{j}=\left(y_{j+1}-y_{j}\right) \exp (-n)$. By mean value theorem, there exists $\chi_{j} \in\left[x_{j}, x_{j+1}\right]$ such that $f^{\prime}\left(\chi_{j}\right)=\left(f\left(x_{j+1}\right)-f\left(x_{j}\right)\right) / \exp (n)$. So, $\left|z_{j}-f^{\prime}\left(\chi_{j}\right)\right| \leq \exp (-i) / 4$ for some $\chi_{j} \in\left[x_{j}, x_{j+1}\right]$, which implies $\left|f^{\prime}\left(\chi_{j}\right)-f^{\prime}\left(x_{j}\right)\right|<\exp (-i) / 4$, and so, $z_{j}$ is at most $\exp (-i) / 2$ far from $f^{\prime}\left(x_{j}\right)$. Let $p_{j}, q_{j} \in \mathbb{N}$ such that $p_{j} \times \exp \left(-q_{j}\right)$ is at most $\exp (-i) / 2$ far from $z_{j}$, hence, at most $\exp (-i)$ far from $f^{\prime}\left(x_{j}\right)$. Observing that the $y_{j}$, and so the $z_{j}$ can be elementarily obtained from $i$ and $j$, the functions $p_{\mathbb{N}}: \mathbb{N}^{2} \rightarrow \mathbb{N}$, and $q_{\mathbb{N}}: \mathbb{N}^{2} \rightarrow \mathbb{N}$ that map $(i, j)$ to corresponding $p_{j}$ and $q_{j}$ are elementarily computable. By Proposition 3, they can be extended to function $p: \mathbb{R}^{2} \rightarrow \mathbb{R}$ and $q: \mathbb{R}^{2} \rightarrow \mathbb{R}$ in $\mathcal{L}$. Consider function $g: \mathbb{R} \times[0,1] \rightarrow \mathbb{R}$ defined on all $(i, x) \in \mathbb{R} \times[0,1]$ by $g(i, x)=p(i, \exp (n) x) \exp (-q(i, \exp (n) x))$. By construction, for $i, j$ integer, we have $g\left(i, x_{j}\right)=p_{j} \exp \left(-q_{j}\right)$.

Consider the function $F$ given by Lemma 5 for function $g$ and $\epsilon: n \mapsto$ $\exp (-n)$. We have $F\left(i, x_{j}\right)=z_{j}$ for all $i, j \in \mathbb{N}$.

For all integer $i$, and all $x \in \mathbb{R}$, we have

$$
\begin{aligned}
\left\|F(i, x)-f^{\prime}(x)\right\| \leq & \left\|F(i, x)-F\left(i,\lfloor x\rfloor_{\epsilon}\right)\right\|+\| F\left(i,\lfloor x\rfloor_{\epsilon}-g\left(i,\lfloor x\rfloor_{\epsilon}\right) \|\right. \\
& +\left\|g\left(i,\lfloor x\rfloor_{\epsilon}\right)-f^{\prime}\left(\lfloor x\rfloor_{\epsilon}\right)\right\|+\left\|f^{\prime}\left(\lfloor x\rfloor_{\epsilon}\right)-f^{\prime}(x)\right\| \\
\leq & \left\|F\left(i,\lfloor x\rfloor_{\epsilon}+\epsilon_{i}\right)-F\left(i,\lfloor x\rfloor_{\epsilon}\right)\right\|+0+\exp (-i)+M \epsilon_{i} \\
\leq & \left\|f^{\prime}\left(x_{j+1}\right)-g\left(i, x_{j+1}\right)\right\|+\left\|f^{\prime}\left(x_{j}\right)-g\left(i, x_{j}\right)\right\| \\
& +\left\|f^{\prime}\left(x_{j+1}\right)-f^{\prime}\left(x_{j}\right)\right\|+\exp (-i)+\exp (-i) / 4 \\
\leq & 3 \times \exp (-i)+\exp (-i)+\exp (-i) / 4 \\
\leq & 5 \times \exp (-i)
\end{aligned}
$$

Consider the function $G: \mathbb{R}^{2} \rightarrow \mathbb{R}$ defined for all $i, x \in \mathbb{R}$ by the linear differential equation $G(i, 0)=f(0)^{7}$ and $\frac{\partial G}{\partial x}(i, x)=F(i, x)$. For all integer $i$, we

\footnotetext{
7 A technique similar to the one we use here to get function $f^{\prime}$, can be used to show that $f(0)$ is always in $\mathcal{L}^{*}$.
} 
have $G(i, 0)-f(0)=0$ and $\left\|\frac{\partial G}{\partial x}(i, x)-f^{\prime}(x)\right\|=\left\|F(i, x)-f^{\prime}(x)\right\| \leq 5 \times \exp (-i)$. By mean value theorem on function $G(i, x)-f(x)$, we get $\|G(i, x)-f(x)\| \leq$ $5 \times \exp (-i)$ on $[0,1]$. Hence, $f(x)$ is the limit of $G(i, x)$ when $i$ goes to $+\infty$ with integer values. We just need to check that schema LIM can be applied to function $G$ of $\mathcal{L}^{*}$ to conclude: indeed, the limit of $G(i, x)$ when $i$ goes to $+\infty$ will exist and coincide with this value, i.e. $f(x)$.

Since $\frac{\partial G}{\partial x}=F$, and hence $\left\|\frac{\partial^{2} G}{\partial i \partial x}\right\|=\left\|\frac{\partial F}{\partial i}\right\|$ and since $\frac{\partial G}{\partial i}=\int_{0}^{x} \frac{\partial F}{\partial i}(i, x) d x$ implies $\left\|\frac{\partial G}{\partial i}\right\| \leq \int_{0}^{1}\left\|\frac{\partial F}{\partial i}\right\| d x \leq\left\|\frac{\partial F}{\partial i}\right\|$ we only need to prove that we can bound $\left\|\frac{\partial F}{\partial i}\right\|$ by $K \times \exp (-i)$ for a constant $K$. But from Lemma 5 , we know that for all $i, x,\left\|\frac{\partial F}{\partial i}(i, x)\right\| \leq 5\left\|g\left(\lfloor i+1\rfloor,\lfloor x\rfloor_{\epsilon_{i}}\right)-g\left(\lfloor i\rfloor,\lfloor x\rfloor \epsilon_{i}\right)\right\|+25 \| g\left(\lfloor i\rfloor,\lfloor x\rfloor \epsilon_{i}+\epsilon_{i}\right)-$ $g\left(\lfloor i\rfloor,\lfloor x\rfloor_{\epsilon_{i}}\right)\|+25\| g\left(\lfloor i+1\rfloor,\lfloor x\rfloor_{\epsilon_{i+1}}+\epsilon_{i+1}\right)-g\left(\lfloor i+1\rfloor,\lfloor x\rfloor_{\epsilon_{i+1}}\right) \|$. First term can be bounded by $5 \times \exp (-i)+5 \times \exp (-i)=10 \times \exp (-i)$. Second term can be bounded by $25\left(\left\|g\left(\lfloor i\rfloor,\lfloor x\rfloor \epsilon_{i}+\epsilon_{i}\right)-f^{\prime}\left(\lfloor x\rfloor_{\epsilon_{i}}+\epsilon_{i}\right)\right\|+\left\|f^{\prime}\left(\lfloor x\rfloor_{\epsilon_{i}}+\epsilon_{i}\right)-f^{\prime}\left(\lfloor x\rfloor_{\epsilon_{i}}\right)\right\|+\right.$ $\left.\left\|g\left(\lfloor i\rfloor,\lfloor x\rfloor_{\epsilon_{i}}\right)-f^{\prime}\left(\lfloor x\rfloor_{\epsilon_{i}}\right)\right\|\right) \leq 25 \times \exp (-i)+25 \times \exp (-i)+25 \times \exp (-i)=$ $75 \times \exp (-i)$. Similarly for third term, replacing $i$ by $i+1$.

Hence $\left\|\frac{\partial F}{\partial i}(i, x)\right\| \leq 160 \times \exp (-i)$, and so schema LIM can be applied on function $G$ of $\mathcal{L}^{*}$ to get function $f$. This ends the proof.

The missing inclusion of Theorem 2 can be proved similarly for all levels $n \geq 3$ of the Grzegorczyk hierarchy.

Proposition 8. Let $f: \mathcal{D} \subset \mathbb{R}^{k} \rightarrow \mathbb{R}^{l}$ be some function over the reals of class $\mathcal{C}^{2}$, with $\mathcal{D}$ product of compact intervals with rational endpoints. If $f$ is $\mathcal{E}_{n}(\mathbb{R})$, for $n \geq 3$, then it belongs to $\mathcal{L}_{n}^{*}$.

Remark 1. - We have actually a normal form theorem: previous proof shows that every function of $\mathcal{L}^{*}$ and $\mathcal{L}_{n}^{*}$ can be defined using only 1 schema LIM.

- A corollary of this remark is that composing several LIM schemata is always equivalent to at most two for functions of our classes.

\section{References}

1. V. I. Arnold. Ordinary Differential Equations. MIT Press, 1978.

2. E. Asarin and A. Bouajjani. Perturbed Turing machines and hybrid systems. In Logic in computer science, pages 269-278, 2001.

3. E. Asarin and O. Maler. Achilles and the tortoise climbing up the arithmetical hierarchy. Journal of Computer and System Sciences, 57(3):389-398, dec 1998.

4. L. Blum, F. Cucker, M. Shub, and S. Smale. Complexity and Real Computation. Springer-Verlag, 1998.

5. O. Bournez. Achilles and the Tortoise climbing up the hyper-arithmetical hierarchy. Theoretical Computer Science, 210(1):21-71, 61999.

6. O. Bournez. Complexité algorithmique des systèmes dynamiques continus et hybrides. PhD thesis, École Normale Supérieure de Lyon, janvier 1999.

7. M. Bowles. United States technological enthusiasm and the british technological skepticism in the age of the analog brain. In IEEE Annals of the History of Computing, volume 4, pages 5-15, 1996. 
8. M. Campagnolo, C. Moore, and J. F. Costa. An analog characterization of the Grzegorczyk hierarchy. Journal of Complexity, 18(4):977-1000, 2002.

9. M. L. Campagnolo. Computational complexity of real valued recursive functions and analog circuits. PhD thesis, Universidade Técnica de Lisboa, 2001.

10. G. Etesi and I. Németi. Non-Turing computations via Malament-Hogarth spacetimes. International Journal Theoretical Physics, 41:341-370, 2002.

11. D. Graça and J. F. Costa. Analog computers and recursive functions over the reals. Journal of Complexity, 19:644-664, 2003.

12. A. Grzegorczyk. Computable functionals. Fundamenta Mathematicae, 42:168-202, 1955.

13. T. Henzinger and J.-F. Raskin. Robust undecidability of timed and hybrid systems. Hybrid systems: computation and control; second international workshop, hscc'99, berg en dal, the netherlands, march 29-31, 1999; proceedings, 1569, 1999.

14. M. L. Hogarth. Does general relativity allow an observer to view an eternity in a finite time? Foundations of physics letters, 5:173-181, 1992.

15. D. Lacombe. Extension de la notion de fonction récursive aux fonctions d'une ou plusieurs variables réelles III. Comptes rendus de l'Académie des Sciences Paris, 241:151-153, 1955.

16. L. Lipshitz and L. A. rubel. A differentially algebraic replacement theorem, and analog computability. Proceedings of the American Mathematical Society, 99(2):367-372, February 1987.

17. C. Moore. Recursion theory on the reals and continuous-time computation. Theoretical Computer Science, 162(1):23-44, 51996.

18. J. Mycka. Infinite limits and R-recursive functions. Acta Cybernetica, 16:83-91, 2003.

19. J. Mycka. $\mu$-recursion and infinite limits. Theoretical Computer Science, 302:123$133,2003$.

20. P. Odifreddi. Classical recursion theory II. North-Holland, 1999.

21. T. Ord. Hypercomputation: computing more than the Turing machine. Technical report, University of Melbourne, september 2002. available at http://www.arxiv.org/abs/math.lo/0209332.

22. P. Orponen. Algorithms, languages and complexity, chapter A survey of continuoustime computational theory, pages 209-224. Kluwer Academic Publishers, 1997.

23. M. B. Pour-El. Abstract computability and its relation to the general purpose analog computer (some connections between logic, differential equations and analog computers). Transactions of the American Mathematical Society, 199:1-28, 1974.

24. H. Rose. Subrecursion: Functions and Hierarchies. Clarendon Press, 1984.

25. C. E. Shannon. Mathematical theory of the differential analyser. Journal of Mathematics and Physics MIT, 20:337-354, 1941.

26. H. Siegelmann. Neural networks and analog computation - beyond the Turing limit. Birkauser, 1998.

27. W. Thomson. On an instrument for calculating the integral of the product of two given functions. In Proceedings of the royal society of London, number 24, pages 266-276, 1876.

28. A. Turing. On computable numbers, with an application to the "Entscheidungsproblem". In Proceedings of the london mathematical society, volume 2, pages 230-265, 1936.

29. K. Weihrauch. Computable Analysis. Springer, 2000.

30. Q. Zhou. Subclasses of computable real valued functions. Lecture Notes in Computer Science, 1276:156-165, 1997. 